Wang, C.M. (2012). An investigation of using wikis as a collaborative tool for teaching in a non-western tertiary education classroom. Journal of Educational Technology Development and Exchange, 5(1), 63-76.

\title{
An Investigation of Using Wikis as a Collaborative Tool for Teaching in a Non-Western Tertiary Education Classroom
}

\author{
Chun-Min Wang \\ Hsinchu University of Education
}

\begin{abstract}
Wikis are innovative Web 2.0 tools that allow users to create, expand, and edit content collaboratively. This makes wikis promising for online collaborative learning, but further exploration is required to determine if using wikis can achieve learning goals efficiently and appropriately. With increasing globalization, it is useful to determine how students from nonWestern cultures respond to using wikis in the learning environment. In this study, the author compares two Taiwanese undergraduate classes with different instructional design and peer assessment strategies to understand Taiwanese student online behaviors and learning preferences in a learning environment using wikis. The results indicate that students prefer to be assigned responsibility in group projects rather than free writing assignments, and student collaboration is limited in the assignment. The author also discusses the efficiency of in-group peer assessment and inter-group peer assessment used in group projects. The author suggests using wikis as a knowledge management system may be better than using wikis as a collaborative tool in tertiary education classroom.
\end{abstract}

Keywords: wikis, Web 2.0, collaboration, peer assessments, Taiwanese students

\section{Introduction}

With the rapid growth of online technology, online education has become critical for higher education institutions' long-term strategies and is no longer a marginal part of the education system, but as a part of the mainstream (Harasim, 2000). To take advantage of new educational trends and online technology, it is important to examine how to appropriately incorporate these online technologies within education. Wikis are popular emerging online technology tools, which have been used for educational purposes in schools (Beldarrain, 2006; Brewer \& Milam, 2006). Researchers and instructors have begun to explore potential wiki applications in the classroom (Ferris \& Hilary, 2006). Not until recently, most discourses concerning wikis were hypothetical statements (Johnson, Hulme, \& Graham, 2007; Kelsey, 2007). Many empirical studies have since been conducted, and several suggestions have been generated for applying wikis to teaching and learning. However, these findings are mostly based on learners from Western cultures. Given globalization trends, it is important to also research students from different cultures. There is also a need of reflection regarding the use of wikis in the classroom after years of educational applications. This study explores wiki learning behaviors in a nonWestern culture and examines the efficiency 
of using wikis by comparing two Taiwanese classes with different instructional designs and assessment strategies. The study also examines the wiki teaching experience and discusses whether wikis are an efficient and appropriate tool for collaborative projects in tertiary education classrooms.

\section{Background}

\subsection{Wiki Potential}

Compared to other Web 2.0 tools, wikis allow users to edit content collaboratively without knowing how to program a Web page. This feature makes wikis the perfect virtual space in which to practice collective knowledge construction. An example of a successful wiki is Wikipedia; a free encyclopedia that allows anyone to contribute to its content. With the success of Wikipedia, teachers and educational researchers have become interested in using wikis in formal educational settings. Most studies have focused on the collaborative capabilities of wikis (Lundin, 2008). Wheeler, Yeomans, and Wheeler (2008) found that student writing skills improved by using wikis as collaborative tools. Learners are also more satisfied with wikis than MS Word in collaborative writing activities (Shu \& Chuang, 2012). Additionally, wikis are an ideal platform for generating reading and writing assignments and can provide an easy method for students to structure discussions outside the classroom (Farabaugh, 2007; Chao \& Lo, 2011). Educators also found that wikis are useful when preparing doctoral qualification examinations for doctoral students with similar research interests because they allow students to share different perspectives (DiPietro et al., 2010). Wikis were also recommended as knowledge management systems, allowing users to consistently update information (King, 2007). The results of these studies clearly indicate that wikis can be used in the classrooms. However, as Laurillard (2009) stated, educational practitioners should not merely adopt what markets and businesses provide, but should think of what is required for learning and challenging new technologies to fulfill the requirements. In other words, although wikis have shown potential for use in educational settings, practitioners must consider whether wikis are really appropriate for student learning. Perhaps wiki applications conflict with student learning preferences, thus creating an inefficient environment that does not achieve desired learning goals.

\subsection{Theoretical Foundation}

Wikis provide an environment for participants to construct content collaboratively and create an online community that involves apparent or ambiguous rules and standards. Thus, to facilitate learning, participants must know what types of social engagements can provide proper context. Lave and Wenger (1991) proposed the notion of communities of practice, which provides insights for researchers to help them understand the wiki learning environment. Lave and Wenger argued that communities of practice are everywhere, and people are generally involved in several communities, whether at school, work, or home. They emphasized connecting "issues of sociocultural transformation with the changing relations between newcomers and old-timers in the context of a changing shared practice" (Lave \& Wenger, 1991, p. 49). A community of practice, as defined by Lave and Wenger (1991) is “...a set of relations among persons, activities, and the world, over time and in relation with other tangential and overlapping communities of practice" (p. 98). A community must have mutual engagement, joint enterprises, and shared repertoires as the source of community coherence (Wenger, 1998). This implies that the community must accept member diversity, and each community participant should have a unique place and identity. Rather than people knowing everything themselves, it is more important to know how 
to collaborate and build mutual relationships in the community. Joint enterprises are the result of negotiation among community participants, and they reflect complex mutual engagement between participants. A shared repertoire is created by pursuing joint enterprises and includes "routines, words, tools, ways of doing things, stories, gestures, symbols, genres, actions, or concepts that the community has produced or adopted in the course of its existence, and which have become part of its practice" (Wenger, 1998, p. 83).

Lave and Wenger (1991) stated that learning is "an evolving continuously renewed set of relations" (p. 50). Lave and Wenger believed that a community of practice is built on a limited number of people with mutual relationships, but Brown and Duguid (2000) thought that networks can link people working on similar practices who do not know each other. Therefore, communities of practice can be extended by networks such as Web sites, bulletin boards, and listservs. Wikis, can thus, be understood and examined using the concept of communities of practice.

\subsection{Application Challenges}

Although wikis have the potential to be used in classrooms, wiki activities may not suit the learning preferences of all students (Wheeler et al., 2008). As Collis and Moonen (2008) indicated, a challenge of using Web 2.0 tools in the classroom is the conflict between pedagogical approaches and student expectations. Unlike traditional classrooms that use a teacher-centered approach, a wiki learning environment usually adopts a more learner-centered approach. The instructor and all students must contribute to and participate in activities to ensure high-quality learning. Without a change in mindset and culture in the classroom, learning outcomes may be unexpected. Twu (2010) indicated that wikis technology is an attitude rather than simply a tool, therefore providing support resources for instructors and students to assess the processes are needed (Collis \& Moonen, 2008). Educating students to value other contributions and negotiate with group members are also important to the success of wiki collaborative assignment (Collis \& Moonen, 2008; Wheeler et al., 2008).

Wikis can create ownership and intellectual property problems. Research has found that students are resistant to having their contributions edited or deleted by other group members (van den Berg et al., 2006). However, wiki use in non-Western cultures does not necessarily have similar constraints. Perhaps, as Twu (2010) stated, educators must first understand student cultural backgrounds to cultivate positive attitudes toward using wikis. Therefore, how students in non-Western cultures react to wiki learning environments should be explored further.

\subsection{Research Questions}

This study addresses these research issues by examining a wiki learning environment in a university in Taiwan. Two classes were compared to answer two research questions. First, how should team collaboration be regulated to motivate students to contribute to the group project? Second, how should individual performance be assessed in a group project? By exploring these two questions, the study attempts to understand student learning preferences in a wiki environment and investigate using wikis as a collaborative tool in a non-Western tertiary education classroom.

\section{Methods}

\subsection{Research Context}

The wiki learning environment was created using an open source wiki package called MediaWiki (http://www.mediawiki.org). Two classes of college freshmen in the Department of Education in a Northern Taiwan university participated in the study. The students were 
enrolled in the same course, "Introduction to Learning Technology," and had the same instructor. Class A had 38 students (7 males), and Class B had 39 students (8 males). The two classes were identical for student academic performance because students were assigned to classes uniformly based on their college entrance scores. The collaborative project was one of the assignments in the course. After the activity, each student was asked to fill out a questionnaire, and twelve students were selected for the follow-up individual interviews for the data collection.

\subsection{Research Design}

For the collaborative project, students were divided into groups of four, and each group was required to write a chapter collaboratively using the wiki platform. For the chapter, each group selected and explored a learning technology and addressed the following four items: (a) the history of the learning technology, (b) its current educational applications, (c) its advantages and disadvantages, and (d) its future trends. Each group had approximately one month to create the chapter, but different strategies regulated the writing and assessment processes in Classes A and B.

Class A (control group): Students were asked to complete the chapter in four weeks. No other regulations were required during the collaborative writing period. Each group decided and negotiated the workload of its group members. At the end of the semester, the instructor graded the final chapter. Each group member received the same score, but individual scores were adjusted based on in-group peer assessments. The in-group peer assessments required students to evaluate the contribution of each group member.

Class B (experimental group): Each team member had a week to work on the chapter, and during that week, other team members could not edit the content. The chapter was then opened for team members editing in the fifth week. The instructor evaluated each team member's performance each week, and other groups in the class assessed the final chapter. A student's final score was the average of their individual performance in their week and the final chapter score graded by the other groups.

\subsection{Data Collection}

This study used two methods of data collection. A questionnaire was distributed at the end of the semester to survey student satisfaction with the wiki learning experience. The questionnaire included twenty 5-point Likert-scale questions that were reviewed by three experts to ensure content validity (see Appendix 1). Follow-up interviews were also conducted right after the questionnaire. Twelve students from Classes A and B were selected to participate in face-to-face interviews. Six students were selected from each class: three from the students with the highest scores and three from the students with the lowest scores. Each semi-structured interview lasted approximately 30 minutes and was audiorecorded. These interviews were all conducted by the same investigator, and an interview protocol developed based on the research questions was used to guide the interviews (see Appendix 2).

\subsection{Data Analysis}

The questionnaire results were transformed into numerical scores, but the scores were on an ordinal scale. Thus, Mann-Whitney U tests were used to compare question responses from Classes A and B. The findings were used to discuss student preferences for using wikis for collaborative projects. The interviews were analyzed using an open-coding strategy to identify the main themes in each question. The results of the interviews were used as additional information to understand student wiki learning experiences. 


\section{Results}

\subsection{Questionnaire}

Class A produced 37 valid samples (of 38), and Class B produced 34 valid samples (of 39). Table 1 shows the mean scores, standard deviations, and results of the Mann-Whitney U tests for each question. The results show that Class B had higher mean scores than Class A for all questions except Questions 7 and 8. This indicates that students preferred the assessment process used in Class A, but Class B students were more satisfied with the wiki collaborative writing experience. The Mann-
Whitney $\mathrm{U}$ tests showed that six questions (Q2, Q4, Q5, Q8, Q16, and Q20) were significantly different between Classes A and $\mathrm{B}$ ( $p \leq$ $.05)$. This confirms that Class B was more satisfied than Class A with the collaborative writing experience, and Class B students were more satisfied with the overall wiki learning experience. However, the results also indicated that Class B students were less satisfied with the assessment process. In other words, the ingroup peer assessments used in Class A were more satisfactory than the inter-group peer assessments used in Class B.

Table 1. Question Results ( $\mathrm{A}=$ Class A, B=Class B).

\begin{tabular}{cccccc}
\hline & A Mean & B Mean & A St. Dev & B St. Dev & $p$-value \\
\hline Q1 & 3.59 & 3.91 & .798 & .668 & .084 \\
Q2 & 3.32 & 3.97 & .884 & .717 & $.002^{* *}$ \\
Q3 & 3.35 & 3.79 & .857 & .914 & .060 \\
Q4 & 3.51 & 4.12 & .768 & .640 & $.001^{* *}$ \\
Q5 & 3.30 & 3.82 & .909 & .797 & $.015^{*}$ \\
Q6 & 3.30 & 3.50 & .812 & .615 & .450 \\
Q7 & 3.59 & 3.50 & .644 & .788 & .758 \\
Q8 & 3.41 & 2.85 & .832 & .958 & $.010^{* *}$ \\
Q9 & 3.50 & 3.53 & .755 & .788 & .959 \\
Q10 & 3.24 & 3.26 & .830 & .898 & .961 \\
Q11 & 3.11 & 3.50 & 1.02 & .749 & .059 \\
Q12 & 3.38 & 3.53 & 1.06 & .825 & .597 \\
Q13 & 3.27 & 3.38 & .932 & .739 & .804 \\
Q14 & 3.35 & 3.65 & 1.03 & .646 & .252 \\
Q15 & 3.16 & 3.65 & 1.17 & .950 & .053 \\
Q16 & 3.24 & 3.91 & .955 & .621 & $.002^{* *}$ \\
Q17 & 3.32 & 3.50 & .944 & .749 & .618 \\
Q18 & 2.86 & 3.15 & 1.00 & .989 & .289 \\
Q19 & 3.43 & 3.76 & .899 & .781 & .085 \\
Q20 & 3.35 & 3.85 & 1.06 & .657 & $.026^{*}$ \\
\hline
\end{tabular}

Note: Class A has 37 responses, and class B has 34 responses.

Criteria alpha $=.05, p$-values are the results of Mann-Whitney $\mathrm{U}$ tests, $*$ denotes $p$-value $\leq .05$, $* *$ denotes $p$-value $\leq .001$ 


\subsection{Follow-up Interviews}

Twelve interviews were conducted at the end of the semester. To present the results in an organized manner, student thoughts and comments were analyzed and summarized into four categories: writing regulations, assessment process, collaborative writing, and course management. Instead of translating the Chinese transcriptions into English and inserting citations, this section presents the interview findings directly.

For the writing regulations, students from both classes preferred taking turns to write the chapter, as required in Class B. They liked being assigned individual responsibility by the instructor instead of negotiating the workload in a group. Although students preferred the regulations used in Class B, students in Class $\mathrm{B}$ mentioned that the last student who was required to write the chapter experienced more stress because there was less content left unwritten.

For the assessment process, although the surveys showed that students were more satisfied with in-group peer assessments than inter-group peer assessments, the interview results revealed a different perspective. Class A students mentioned that in-group peer assessments did not reflect the truth. For example, they did not want their teammates to receive bad scores even if they contributed little or nothing, because they were not only classmates, but also friends or roommates. Therefore, the objectivity of the in-group peer assessments is questionable, and some Class A interviewees emphasized that scores should better reflect efforts. However, the student chosen as contributing the most to a group was relatively consistent among group members. From this perspective, in-group peer assessments are relatively objective. By contrast, interviewees reported that inter-group peer assessments were too subjective because only one or two team members participated in grading. The scores did not emerge from a thorough discussion among team members as originally planned. In addition, because nine groups were graded, students seemed to become more careless toward the end of the group presentations.

When asked of the collaborative writing process, students from both classes mentioned that they did not revise what others had written because they did not want to offend group members and thought it was impolite to do so. Students also thought that their revisions may be incorrect because they are not content experts. Another common phenomenon was that students usually left working on the assignment to the last minute. Consequently, they did not value the opportunity to collaborate with team members, but instead complained that more time was required. Students also tended to complete their own work and then did not want to do more. An interviewee from Class B said that she did not revise the content during the fifth week. Interviewees also mentioned that some students in Class A did not do their assigned sections. Thus, to avoid receiving a bad grade, diligent students wrote content for other teammates. Students first wrote the chapters in Word documents and then posted them to the wiki pages because wiki editing requires special syntax. If students forgot to save the content, they had to rewrite it.

For course management, Class A students thought the assignment could be done individually because everyone completed their parts individually. Collaboration only occurred when sharing information with other teammates. They also suggested implementing strategies to motivate team members who did not contribute. By contrast, Class B students focused on improving the assessment process. They suggested allowing more time for intergroup peer assessments and also indicated 
that scores were informed by presentation skills rather than chapter quality, as intended. Classes A and B preferred the instructor to assign responsibilities and topics for the assignment.

\section{Discussion}

\subsection{Wiki Learning Behaviors}

This study intended to use wikis to create an online environment where students could easily write collaborative chapters and learn from each other. However, no students revised others' writing and little discussion occurred during collaboration. They thought it was impolite and incorrect to change what others wrote and did not like others changing their writing. This may be because of the Confucian heritage that emphasizes group harmony in Chinese culture (Xiao \& Lucking, 2008). Students are nurtured to respect others and save face for everyone. However, compared to research from other cultures (Grant, 2009; van den Berg et al., 2006), this phenomenon also occurs. The unwillingness to change what others have written seems to be common in a wiki-based learning environment. Therefore, how can instructors create efficient learning by using wikis? Successful cases of using wikis, such as in preparing for doctoral qualification examinations (DiPietro et al., 2010), occur when students are highly motivated, are under no grading pressure, and are willing to help others. Therefore, if these requirements are absent, creating a successful learning experience in a wiki-based learning environment is difficult.

Working as a team did not create peer pressure to maintain consistent writing. Instead, social loafing occurred among members and undermined the collaborative project. In an extreme example from Class A, the whole chapter was written by one person because other team members were irresponsible and were not motivated to complete the assignment. To ensure fair individual grades, appropriate assessment strategies are thus important.

\subsection{Peer Assessments}

Assessing individual performance in group projects is difficult. Trentin (2009) proposed using the wiki tracking function and formulas to calculate individual grades. The strategy is creative, but as Grant (2009) argued, "focusing separately on the contributions of individual members may not adequately reflect the collaborative nature of the learning that has taken place" (p. 114). Therefore, although focusing on quantitative data such as words written, feedback given, or revisions may seem logical and fair, it may misunderstand the educational process and encourage utilitarianism in the classroom. Instructors should focus more on the learning process than the final grading process, and students should also be educated in this manner. This study used peer assessments as part of the grading process because peer assessments can provide a relatively objective opinion of the collaboration process. Although this study identified problems with in-group and inter-group peer assessment, techniques could be used to improve outcomes such as anonymous in-group peer assessment or requiring all students to participate in inter-group peer assessment. To exploit both approaches, assignments could use both in-group and inter-group peer assessments with explicit requirements for appropriate evaluation of individual performance in group projects.

\subsection{Learning Preferences}

Students prefer instructors to explicitly regulate assignments and prefer to work on assignments individually. They would 
rather have individual responsibility and an individual grade. They even prefer writing in separate paragraphs to make it easier for the instructor to identify their contributions. This created challenges when Class A students were required to negotiate responsibilities in groups and were assigned a single group score. Interviewees mentioned that Class A students also divided the whole chapter into parts and each student was individually responsible for their part. This is similar to what Zhang, Peng, and Hung (2009) found in their study that Taiwanese students view cooperation as collaboration. Rather than having a mutual engagement throughout the process, Taiwanese students prefer a division of role and labor during group projects. This reveals student learning preferences, but also identifies how to improve group collaboration. Because using wikis in collaboration requires conceptual change (Twu, 2010), time is necessary to value group collaboration and teaching skills are required to guide and facilitate the process. Without collaboration skills and motivation to use wikis, it is difficult to create a successful collaborative learning (Witney \& Smallbone, 2011). Additionally, as found by Allen and Tay (2012) wikis are valuable for individual students to develop their ideas, thus perhaps wikis are better to be used as individual tool than as a group collaborative tool.

\subsection{Instructional Design}

Instructional design is also essential for using wikis in the classroom. In this study, the goal was to design a collaborative project for students to use wikis and learn how to collaborate and view things from teammate perspectives. However, based on the results and instructor observations, little discussion and collaboration among team members actually occurred. Some students even plagiarized from the Internet. Because students have different writing styles, chapters also appeared fragmented. Piezon and Donaldson (2005) suggested possible strategies to manage social loafing such as clarifying roles and responsibilities, making tasks more meaningful for individual student, emphasizing the importance of teamwork, limiting group numbers to the minimum required to accomplish group goals, and requiring high levels of accountability. These strategies are helpful for improving the design and implementation of a collaboration project.

From a community of practice perspective, students in this project were required to develop new collaborative practices from nothing in a relatively short period. This is different from Wikipedia, because many practices already exist for outsiders to observe and learn from. To use wikis during a semesterlong (or shorter) collaborative project, the instructor must provide samples and allow students time to form their communities of practice. More time is suggested for students grounded in Chinese heritage (Xiao \& Lucking, 2008), and more training is needed for them to develop self guidance in less structured online learning environment (Zhang, Peng, \& Hung, 2009). Although strategies, such as educating students to value other contributions and group member negotiation, are suggested (Collis \& Moonen, 2008), it was difficult to change student wiki behaviors, although students were reminded several times. This study suggests the following:

- For course assignments, using wikis for recording individual writing progress and allowing others to add comments are more efficient than group collaborative writing.

- Wikis could be used in group collaborative writing when the exercise is non-graded and affiliated because it is easier to activate student intrinsic motivations. 


\section{Conclusion}

This study found that using wikis for a collaborative writing assignment in a college classroom was not as efficient as originally hypothesized. This is mainly because of conflict between student learning preferences and assignment design. Students tended to divide the collaborative assignment into individual parts and only take responsibility for their own parts. They did not revise what others wrote; thus, little collaboration occurred. Similar results have been found by studies conducted in Western cultures, indicating that this phenomenon is crosscultural. This encourages educators to examine what is required for learning, rather than examining how to use wikis to facilitate learning. Students can collaborate without using wikis, and wikis should be used for collaborative projects because they facilitate and improve learning. If not, educators should reconsider whether using wikis is appropriate and efficient. Although researchers have suggested methods of educating students to respect revisions and value other contributions, in reality these are inefficient teaching approaches. From a community of practice perspective, mutual engagement, joint enterprises, and shared repertoires are the source of community coherence (Wenger, 1998). However, it is difficult to build such an online community in the short 4- or 5-week period used in this study. Therefore, this study recommends that educators carefully examine course goals to maintain learning quality when using wikis as collaborative tools.

Although wikis may not be efficient collaborative teaching tools, the virtual space provides a good platform for individual records of writing progress. Based on student learning preferences and online behaviors, using wikis as personal knowledge management systems that allow others to contribute may be better than using wikis as collaborative tools for group projects. This study also recommends adopting peer assessment strategies to evaluate individual performance in group projects because these strategies can be used to evaluate invisible learning processes and visible learning outcomes and help establish a sense of

\section{References}

Allen, M., \& Tay, E. (2012). Wikis as individual student learning tools: The limitations of technology. International Journal of Information \& Communication Technology Education, 8(2), 61-71. doi: 10.4018/jicte. 2012040105

Beldarrain, Y. (2006). Distance education trends: Integrating new technologies to foster student interaction and collaboration. Distance Education, 27(2), 139-153.

Brewer, S., \& Milam, P. (2006). SLJ's technology survey 2006: New technologies--Like blogs and wikis--Are taking their place in the school media center. School Library Journal, 52(6), 46-50.

Brown, J. S., \& Duguid, P. (2000). The social life of information. Boston: Harvard Business School Press.

Chao, Y.-C. J., \& Lo, H.-C. (2011). Students' perceptions of Wiki-based collaborative writing for learners of English as a foreign language. Interactive Learning Environments, 19(4), 395-411. doi: 10.10 80/10494820903298662

Collis, B., \& Moonen, J. (2008). Web 2.0 tools and processes in higher education: Quality perspectives. Educational Media International, 45(2), 93-106.

DiPietro, J. C., Drexler, W., Kennedy, K., Buraphadeja, V., Feng, L., \& Dawson, K. (2010). Using wikis to collaboratively prepare for qualifying examinations. TechTrends: Linking Research \& Practice 
to Improve Learning, 54(1), 25-32. doi: 10.1007/s11528-009-0360-0

Farabaugh, R. (2007). 'The isle is full of noises': Using Wiki software to establish a discourse community in a Shakespeare classroom. Language Awareness, 16(1), 41-46.

Ferris, S. P., \& Hilary, W. (2006). Uses and potentials of Wikis in the classroom. Journal of online education, 2(5).

Grant, L. (2009). 'I DON'T CARE DO UR OWN PAGE!' A case study of using wikis for collaborative work in a UK secondary school. Learning, Media \& Technology, 34(2), 105-117. doi: 10.1080/1743988090 2923564

Harasim, L. (2000). Shift happens: Online education as a new paradigm in learning. Internet and Higher Education, 3, 41-61.

Johnson, C., Hulme, S., \& Graham, C. R. (2007, October). To Wiki or not to Wiki: That is the question. Paper presented at the annual meeting of the Association for Educational Communications and Technology, Anaheim, CA.

Kelsey, K. (2007, October). Page editing behavior and collaborative learning in a Wikibook project. Paper presented at the annual meeting of the Association for Educational Communications and Technology, Anaheim, CA.

King, W. R. (2007). IT strategy and innovation: Recent innovations in knowledge management. Information Systems Management, 24(1), 91-93.

Laurillard, D. (2009). The pedagogical challenges to collaborative technologies. International Journal of Computer-Supported Collaborative Learning, 4(1), 5-20.

Lave, J., \& Wenger, E. (1991). Situated learning: Legitimate peripheral participation. Cambridge England ; New York: Cambridge University Press.

Lundin, R. W. (2008). Teaching with wikis: Toward a networked pedagogy.
Computers \& Composition, 25(4), 432-448. doi: 10.1016/ j.compcom.2008.06.001

Piezon, S. L., \& Donaldson, R. L. (2005). Online groups and social loafing: Understanding student-group interactions. Online Journal of Distance Learning Administration, 8(4). Retrieved from http://www.westga.edu/ distance/ojdla/ winter84/piezon84.htm

Shu, W., \& Chuang, Y.-H. (2012). Wikis as an effective group writing tool: A study in Taiwan. Online Information Review, 36(1), 89-103. doi: 10.1108/14684521211 206980

Trentin, G. (2009). Using a wiki to evaluate individual contribution to a collaborative learning project. Journal of Computer Assisted Learning, 25(1), 43-55. doi: 10.1111/j.1365-2729.2008.00276.x

Twu, H.-L. (2010). A predictive study of wiki interaction: Can attitude toward wiki predict wiki interaction in high-context cultures groups? Journal of Educational Technology Development \& Exchange, 3(1), 57-68.

van den Berg, I., Admiraal, W., \& Pilot, A. (2006). Designing student peer assessment in higher education: Analysis of written and oral peer feedback. Teaching in Higher Education, 11(2), 135-147.

Wenger, E. (1998). Communities of practice: Learning, meaning, and identity. Cambridge, U.K. ; New York: Cambridge University Press.

Wheeler, S., Yeomans, P., \& Wheeler, D. (2008). The good, the bad and the wiki: Evaluating student-generated content for collaborative learning. British Journal of Educational Technology, 39(6), 987-995.

Witney, D., \& Smallbone, T. (2011). Wiki work: Can using wikis enhance student collaboration for group assignment tasks? Innovations in Education \& Teaching 
International, 48(1), 101-110. doi: 10.108 0/14703297.2010.543765

Xiao, Y., \& Lucking, R. (2008). The impact of two types of peer assessment on students' performance and satisfaction within a Wiki environment. The Internet and Higher Education, 11(3-4), 186-193. doi: 10.1016/j.iheduc.2008.06.005

Zhang, K., Peng, S. W., \& Hung, J.-L. (2009). Online collaborative learning in a project-based learning environment in Taiwan: A case study on undergraduate students' perspectives. Educational Media International, 46(2), 123-135. doi: 10.10 $80 / 09523980902933425$ 


\section{Appendix 1: Questionnaire}

(Likert five-point scale: 1 very disagree, 2 disagree, 3 neutral, 4 agree, 5 very agree)

\section{Section A: Teamwork}

1. I am satisfied with how the instructor regulates the teamwork.

2. I am satisfied with the way our team share the workload.

3. I am satisfied with my contribution to the chapter.

4. I am satisfied with other team members' contribution to the chapter.

5. I am satisfied with the interactions among team members during the collaborative writing.

\section{Section B: Evaluation}

6. I am satisfied with how the instructor evaluates my performance.

7. I am satisfied with the way I evaluate other teams or team members.

8. I am satisfied with how the other classmates evaluate my performance.

9. I prefer to have individual score rather than one team score for everyone.

10. Generally speaking, I am satisfied with the strategy used to evaluate our performance.

\section{Section C: Wiki platform}

11. I have no problem to learn how to write and edit in wiki environment.

12. I am satisfied with using wiki for learning.

13. I am satisfied with the functions provided in wiki environment.

14. I am satisfied with the information provided on Wikipedia.

15. I am satisfied with my wiki learning experience.

\section{Section D: Overall experience}

16. I am satisfied with the collaborative writing experience.

17. I am satisfied with the instructor's guidance in this assignment.

18. I am satisfied with the time arrangement for collaborative writing. 
19. I am satisfied with what I learned during the collaborative writing process.

20. Generally speaking, I am satisfied with this learning experience.

\section{Appendix 2: Semi-structured interview protocol}

1. How did you arrange your time while working on the chapter?

2. What do you like most about the wiki collaborative writing platform? Why?

3. What do you like least about the wikis collaborative writing platform? Why?

4. What recommendations can you give regarding writing collaboratively?

5. What recommendations can you give regarding the evaluation of performance?

6. Overall, what are you opinions of applying wikis into learning 


\section{Contact the Author}

\section{Chun-Min Wang}

Hsinchu University of Education

Email: cwang@mail.nhcue.edu.tw 